\title{
Effect of Cholesterol Feeding and Pantethine Treatment on Complexes between Lipids and Arterial Comnective Tissue Macromolecules in Specific Pathogen-Free Rabbits
}

\author{
Akio Noma $^{1, *}$ and Yoji Yoshida ${ }^{2}$ \\ ${ }^{1}$ Division of Clinical Biochemistry, Tokyo Metropolitan Geriatric Hospital, \\ Sakae-cho, Tokyo 173, Japan \\ ${ }^{2}$ The First Department of Pathology, Yamanashi Medical College, \\ Tamaho-cho, Yamanashi 490-38, Japan
}

(Received June 16, 1986)

\begin{abstract}
Summary The present study was designed to clarify the significance of complex formation of lipoproteins with extracellular connective tissue macromolecules during lipid accumulation in the arterial wall in SPF rabbits fed on a $0.5 \%$ cholesterol diet for six weeks. The effect of pantethine, a new hypolipidemic drug, on their complex formation and lipid accumulation was also studied. The levels of cholesterol bound to glycosaminoglycan, collagen and elastin in the aortic tissues were significantly increased in the atherogenic-diet group. The present results suggest the importance of complex formation of lipoproteins with elastin for lipid accumulation in atherosclerotic lesions. From the results on the ratios of oleic acid/linoleic acid in cholesteryl esters in the plasma and aortic elastin fractions, the cholesteryl esters accumulated on the connective tissue macromolecules in the atherosclerotic rabbits might be derived from the plasma directly, in addition to vascular cell-derived cholesteryl esters. Treatment with pantethine significantly reduced the increased levels of cholesterol in the plasma and the aortic tissues of the cholesterol-fed rabbits. The results suggest that pantethine acts directly on the arterial tissues, in addition to its hypolipidemic effect. These biochemical results were confirmed by the histological findings.
\end{abstract}

Key Words: cholesterol feeding, pantethine, lipoprotein complex, aorta, intimal thickening

* To whom correspondence should be addressed. 
The interaction of certain plasma lipoproteins with connective tissue macromolecules, such as glycosaminoglycan [1, 2], collagen [3], and elastin [4-6], of blood vessels is considered one of the mechanisms of lipid accumulation in atherosclerotic lesions.

Earlier in vivo studies on lipoprotein-glycosaminoglycan or -elastin interactions were confined mainly to atherosclerotic lesions from humans. However, in view of the limitation of using human aortas, there is a need for an experimental model to study under controlled conditions the influence of the arterial extracellular matrix on lipoprotein flux and accumulation.

In order to obtain further insight into the role of the extracellular connective tissue macromolecules in the association of lipids with the aortic intima-media, the present study was undertaken to explore biochemically and histologically the distribution of lipid complexes among these macromolecules from the aortas of rabbits fed normal and cholesterol-rich diets. Also, the effect of the oral administration of pantethine, an intermediate precursor of coenzyme $\mathrm{A}$ and a new interesting hypolipidemic drug [7-9], was examined in these hyper-cholesterolemic rabbits. The present study was carried out using specific pathogen-free (SPF) male New Zealand white rabbits, placed in individual cages in a barrier system, in order to minimize the differences in strains and conditions.

\section{MATERIALS AND METHODS}

Materials. Chromatographically purified collagenase (400 U/mg dry wt., Clostridium histolyticum) was obtained from Worthington Biochemical Corp. (Freehold, NJ). Porcine pancreatic elastase (70 U/mg dry wt., Type III) and soybean trypsin inhibitor were purchased from Sigma Chemical Co. (St. Louis, MO). D-Glucuronic acid, sodium salt, was obtained from Aldrich Chemical Co. (Milwaukee, WI). All the other chemicals used were the highest available grade.

Animals and diets. Male SPF New Zealand white rabbits weighing $1.5-2.0 \mathrm{~kg}$ were randomly divided into three groups. They were housed individually in a barrier system (temperature, $22 \pm 0.5^{\circ} \mathrm{C}$; humidity, $55 \pm 5 \%$ ) at Japan Laboratory Animals, Inc., Tokyo and prefed for one week with sterilized commercial rabbit chow (Oriental RC-4, Oriental Yeast Co., Tokyo). The control rabbits $(N=8)$ were maintained on $100 \mathrm{~g}$ of the sterilized commercial rabbit chow per day. The atherogenic-diet group $(N=8)$ was fed with the same rabbit pellets supplemented with $0.5 \%$ cholesterol and $5 \%$ peanut oil (100 g/day). Rabbits in the pantethinetreated group $(N=8)$ were maintained on the same diet as the atherogenic-diet group except the pellets also contained $0.6 \%$ pantethine (Daiichi Seiyaku Co., Ltd., Tokyo) (100 g/day).

At the end of a 6-week period, the animals were anesthetized with sodium pentobarbital, sacrificed by bleeding, and the entire aortas were quickly excised. At the time of killing, blood samples were drawn for lipid analyses.

Aortic tissues. The aortas were stripped of all extraneous tissues, slit longitu- 


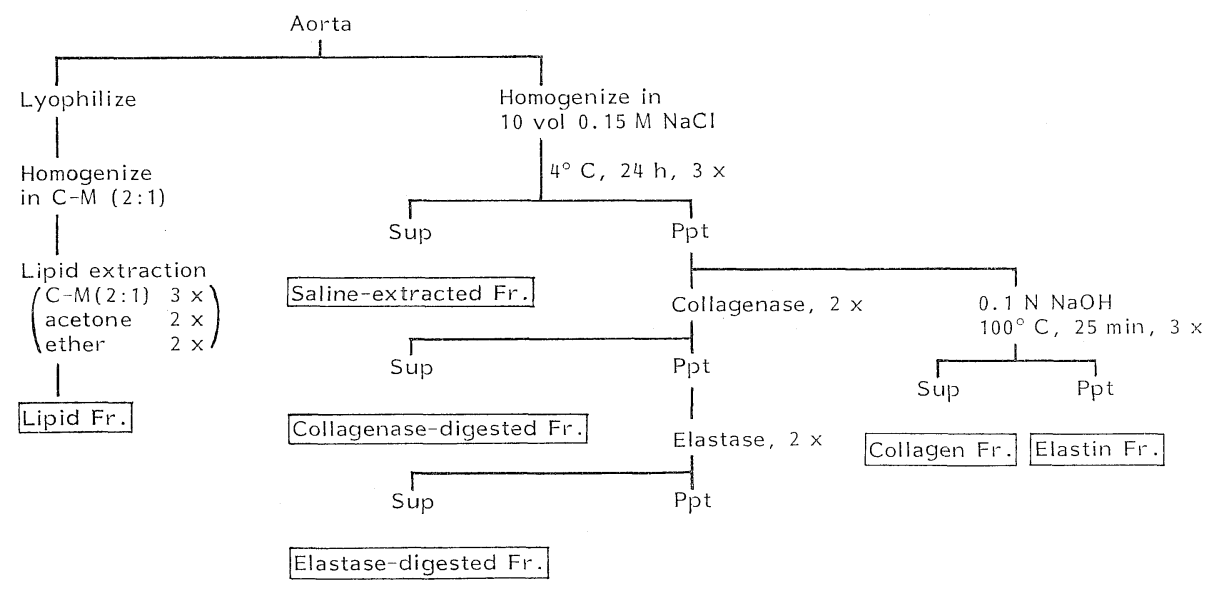

Fig. 1. Procedures of fractionation of lipid and connective tissue macromolecules from rabbit aortas. C, Chloroform; M, methanol; Sup, supernatant; Ppt, precipitate; Fr., fraction.

dinally, rinsed with physiological saline, blotted free of water, and weighed. No attempt was made to separate intima from media or lesion from non-lesion areas in the present study. Sections from the four standard sites (aortic arch, upper and lower thoracic aorta, and abdominal aorta) of aortas of two rabbits, who showed the highest and lowest plasma cholesterol concentrations in each group, were taken for the histological examinations.

Aortic tissues were cut into small pieces, weighed, and divided into two parts which were extracted by the following procedures (Fig. 1):

Lipid fraction. Small pieces of aortas for Lipid fraction were weighed and lyophilized. Lyophilized aortic pieces were homogenized with five volumes of chloroform-methanol $(2: 1, \mathrm{v} / \mathrm{v})$, and extracted successively three times with chloroform-methanol, twice with acetone, and then twice with diethyl ether.

Glycosaminoglycan fractions. A sequential extraction procedure for lipoprotein-glycosaminoglycan complexes was carried out described by Srinivasan et al. [10]. The aortas were minced thoroughly and extracted with 10 volumes of $0.15 \mathrm{M} \mathrm{NaCl}, \mathrm{pH} 7.4$, for $14 \mathrm{~h}$ at $4^{\circ} \mathrm{C}$. After centrifugation, the supernatants were collected and the residues were washed with three volumes of $0.05 \mathrm{M}$ Tris- $\mathrm{HCl}$ buffer, $\mathrm{pH} 7.5$, containing $0.36 \mathrm{~mm} \mathrm{CaCl}_{2}$. The tissues were then digested twice with collagenase ( $150 \mathrm{U} / \mathrm{g}$ original wet tissue) at $37^{\circ} \mathrm{C}$ for $12 \mathrm{~h}$ in five volumes of the above-mentioned Tris- $\mathrm{HCl}$ buffer system and the supernatant collected. The residual tissues were washed twice with $0.2 \mathrm{M}$ Tris- $\mathrm{HCl}$ buffer, $\mathrm{pH} 8.8$, then digested twice with elastase $(60 \mathrm{U} / \mathrm{g}$ original wet tissue) containing soybean trypsin inhibitor (enzyme: inhibitor ratio, $1: 4$ ) at $37^{\circ} \mathrm{C}$ for $12 \mathrm{~h}$ and the supernatant collected. The supernatants from all extractions including washings, defined as Saline-extracted fraction, Collagenase-digested fraction and Elastase-digested fraction, respectively, were extensively dialyzed against $0.15 \mathrm{M} \mathrm{NaCl}$, pH 7.4. 
Collagen and Elastin fractions. A part of the residue after extraction with saline was suspended in 10 volumes of $0.1 \mathrm{~N} \mathrm{NaOH}$ and heated for $25 \mathrm{~min}$ in a boiling water-bath with constant shaking. After cooling and centrifugation, the supernatants, defined as Collagen fraction, was collected. The residue was extracted twice more with $0.1 \mathrm{~N} \mathrm{NaOH}$ and the final residue, defined as Elastin fraction, was successively delipidated as described previously $[6,11,12]$.

Chemical analyses. Whole plasma and Lipid fraction were analyzed for cholesterol, triglyceride and free fatty acids [13] by enzymatic methods with the use of commercial kits, and for phospholipid by Bartlett's method [14]. Cholesterol contents of Glycosaminoglycan fractions, neutralized Collagen fraction and lipids extracted from the Elastin fraction were determined by the enzymatically. Uronic acid was measured by a modification of the carbazol procedure of Bitter and Muir [15], and hydroxyproline, by a modification [16] of Bergman and Loxley's method [17].

For fatty acid analyses, silicic acid column chromatography and thin-layer chromatography were used to isolate cholesteryl ester fractions. The fatty acid composition of cholesteryl esters was determined by gas-liquid chromatographic analysis of fatty acid methyl esters.

Preparation for morphologic examinations. From aortas of two rabbits of each group (control, cholesterol-fed, and cholesterol-fed/pantethine-treated), small samples were taken from each of the four standard sites (aortic arch, upper and lower thoracic aorta, and abdominal aorta). They were fixed in $10 \%$ neutralized buffered-formalin. Following fixation, each sample was subdivided into two segments, one to be paraffin-embedded and the other frozen. The paraffin-embedded sections were stained with hematoxylin and eosin, Weigert's stain for elastic fiberMasson's trichrome, Azan-Mallory stain, periodic acid-Schiff, or alcian blue. The frozen sections were stained with oil red $\mathrm{O}$ for lipids.

\section{RESULTS}

\section{Body weight}

All rabbits steadily gained weight throughout the experimental period. At the end of the sixth weeks, the body weights (mean $\pm \mathrm{SD}$ ) of animals of the control, cholesterol-fed, and cholesterol-fed/pantethine-treated groups were $2.83 \pm 0.04$, $2.81 \pm 0.11$, and $2.82 \pm 0.11 \mathrm{~kg}$, respectively.

\section{Plasma lipids}

The concentrations of lipids in the plasma from the rabbits of the three groups at the end of the experimental period are presented in Table 1. Plasma cholesterol levels were significantly increased 30-40-fold in the cholesterol-fed group and 15-20-fold in the cholesterol-fed/pantethine-treated group over the level in the control rabbits. Mean values with SD of plasma cholesterol concentrations of the three groups throughout the cholesterol feeding period are shown in Fig. 2. As 
Table 1. Effect of cholesterol feeding and pantethine treatment on plasma lipids in male specific pathogen-free New Zealand white rabbits.

\begin{tabular}{lccc}
\hline & Control & Cholesterol-fed & Pantethine-treated \\
\hline No. of animals & 8 & 8 & 8 \\
Cholesterola & $49 \pm 10$ & $1,678 \pm 341^{*}$ & $740 \pm 171^{*} \dagger$ \\
Triglyceride $^{\mathrm{a}}$ & $82 \pm 12$ & $92 \pm 16$ & $77 \pm 20$ \\
Phospholipids $^{\mathrm{a}}$ & $61 \pm 8$ & $509 \pm 43^{*}$ & $277^{*} \pm 54^{*} \dagger$ \\
Free fatty acids $^{\mathrm{b}}$ & $734 \pm 132$ & $894 \pm 238$ & $714 \pm 82$ \\
\hline
\end{tabular}

Values are means $\pm \mathrm{SD}$. a Expressed as $\mathrm{mg}$ per $100 \mathrm{ml}$ of plasma. b Expressed as $\mu \mathrm{mol}$ per liter of plasma. ${ }^{*} p<0.001$ vs. control group, $\dagger p<0.001$ vs. cholesterol-fed group.

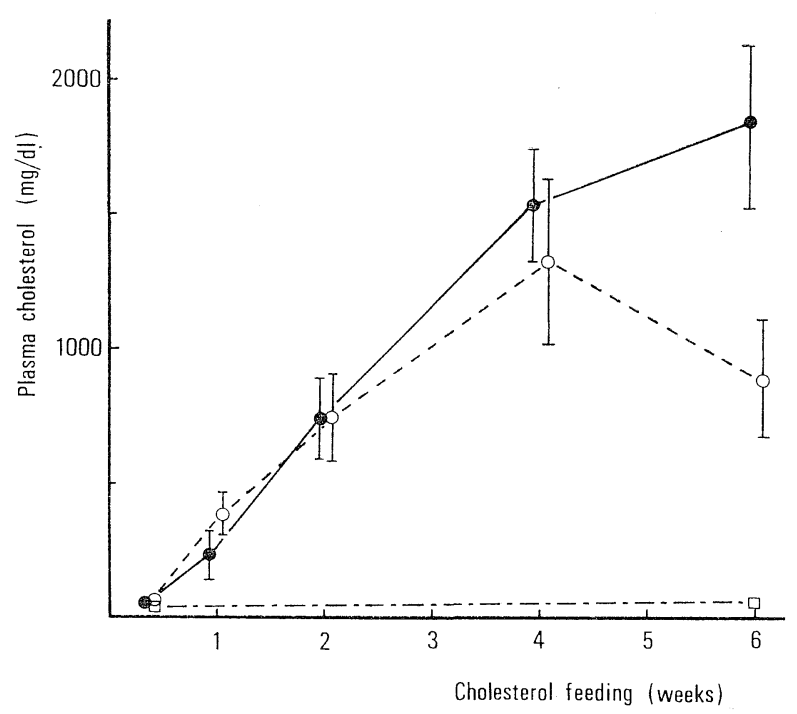

Fig. 2. Time course of plasma cholesterol concentrations of the control, cholesterol-fed and cholesterol-fed/pantethine-treated rabbits during cholesterol feeding. $\square$, Control group; cholesterol-fed group; $\bigcirc$, pantethine-treated group.

shown in the figure, the continuous increase of plasma cholesterol in both experimental groups was almost linear with respect to the time of cholesterol feeding up to 4 weeks. Although plasma cholesterol levels were not significantly different between the experimental groups up to that time, they became significantly different $(p<0.001)$ by 6 weeks on the diet.

Plasma levels of phospholipids were also significantly increased in the experimental rabbits. In contrast, although plasma levels of triglyceride and free fatty acids tended to increase in the cholesterol-fed group, the differences were not statistically significant.

Aortic tissues

Biochemical findings. Cholesterol, triglyceride, and phospholipid conVol. 1, No. 3, 1986 
Table 2. Lipid contents of aortas from the control, cholesterol-fed, and cholesterol-fed/pantethine-treated rabbits.

\begin{tabular}{|c|c|c|c|}
\hline & Control & Cholesterol-fed & Pantethine-treated \\
\hline No. of animals & 5 & 6 & 6 \\
\hline Total cholesterola & $7.2 \pm 0.5$ & $16.6 \pm 2.1^{* * *}$ & $10.7 \pm 1.0^{*} \dagger$ \\
\hline Free cholesterola & $4.8 \pm 0.5$ & $6.7 \pm 0.6^{*}$ & $6.3 \pm 0.7$ \\
\hline Cholesteryl estera & $2.4 \pm 0.2$ & $9.9 \pm 1.6^{* *}$ & $4.4 \pm 0.6 *$, \\
\hline Ester/free ratio & $0.5 \pm 0.1$ & $1.5 \pm 0.2^{* *}$ & $0.7 \pm 0.1 \pm$ \\
\hline Triglyceride ${ }^{a}$ & $8.1 \pm 1.1$ & $13.3 \pm 1.6^{*}$ & $11.0 \pm 1.7$ \\
\hline Phospholipidsa & $31.0 \pm 3.1$ & $36.9 \pm 6.1$ & $33.3 \pm 6.3$ \\
\hline
\end{tabular}

Table 3(A). Cholesterol, uronic acid, and hydroxyproline contents.

\begin{tabular}{|c|c|c|c|}
\hline & Control & $\begin{array}{c}\text { Cholesterol-fed } \\
\text { (mg/g dry defatted tissue) }\end{array}$ & Pantethine-treated \\
\hline \multicolumn{4}{|l|}{ Saline-extracted fraction } \\
\hline Cholesterol & $3.80 \pm 0.33$ & $7.55 \pm 0.81 * *$ & $5.13 \pm 0.59^{*, \dagger}$ \\
\hline Uronic acid & $4.60 \pm 0.45$ & $5.36 \pm 0.51$ & $4.73 \pm 0.58$ \\
\hline Hydroxyproline & $0.42 \pm 0.08$ & $0.56 \pm 0.10$ & $0.51 \pm 0.10$ \\
\hline \multicolumn{4}{|c|}{ Collagenase-digested fraction } \\
\hline Cholesterol & $1.20 \pm 0.11$ & $2.32 \pm 0.29 * *$ & $1.47 \pm 0.33 \dagger$ \\
\hline Uronic acid & $1.45 \pm 0.08$ & $2.23 \pm 0.22 *$ & $1.91 \pm 0.33$ \\
\hline Hydroxyproline & $35.5 \pm 2.4$ & $41.7 \pm 6.5$ & $34.8 \pm 4.3$ \\
\hline \multicolumn{4}{|l|}{ Elastase-digested fraction } \\
\hline Cholesterol & $2.31 \pm 0.19$ & $5.80 \pm 0.46^{* *}$ & $4.14 \pm 0.38 * *, \dagger$ \\
\hline Uronic acid & $1.64 \pm 0.14$ & $2.13 \pm 0.29$ & $2.02 \pm 0.23$ \\
\hline Hydroxyproline & $6.23 \pm 0.61$ & $6.38 \pm 1.09$ & $6.05 \pm 1.00$ \\
\hline \multicolumn{4}{|c|}{ Table 3(B). Cholesterol and connective tissue macromolecules. } \\
\hline \multicolumn{4}{|l|}{ Collagen fraction } \\
\hline Cholesterol & $2.10 \pm 0.20$ & $5.14 \pm 0.69 * *$ & $3.75 \pm 0.46$ \\
\hline Collagena ${ }^{a}$ & $222 \pm 10$ & $224 \pm 42$ & $221 \pm 31$ \\
\hline Cholesterol/collagen ${ }^{\mathrm{b}}$ & $9.5 \pm 0.5$ & $23.5 \pm 3.8 * *$ & $17.4 \pm 3.7^{*}$ \\
\hline \multicolumn{4}{|l|}{ Elastin fraction } \\
\hline Cholesterol & $1.59 \pm 0.16$ & $3.87 \pm 0.42^{* *}$ & $1.75 \pm 0.46$ \\
\hline Elastin & $462 \pm 26$ & $410 \pm 43$ & $414 \pm 30$ \\
\hline Cholesterol/elastin ${ }^{\mathrm{b}}$ & $3.4 \pm 0.3$ & $9.4 \pm 0.5^{*}$ & $4.1 \pm 0.5$ \\
\hline
\end{tabular}

Values are means $\pm \mathrm{SD}$. a Collagen $=$ hydroxyproline $\times 8 . \mathrm{b} \times 1,000 . * p<0.01, * * p<0.001$ vs. control group, $\dagger p<0.01, \ddagger p<0.001$ vs. cholesterol-fed group.

centrations of the Lipid fraction from the aortas of the control, cholesterol-fed, and cholesterol-fed/pantethine-treated rabbits are shown in Table 2. Cholesterol levels in the aortic tissues of both experimental groups were significantly different from those of the control group. It is clear from this table that the increase of cholesteryl ester contents were primarily responsible for the differences seen in total cholesterol concentrations among these groups. On the other hand, aortic 
Table 4. Oleic acid to linoleic acid ratios in plasma cholesteryl esters and in cholesteryl esters bound to aortic elastin fraction.

\begin{tabular}{lllc}
\hline & Control & Cholesterol-fed & Pantethine-treated \\
\hline Plasma cholesteryl esters $^{\mathrm{a}}$ & $0.42 \pm 0.06$ & $1.48 \pm 0.09^{* * *}$ & $1.27 \pm 0.03^{* * \dagger}+$ \\
Cholesteryl esters bound to elastin $^{\mathrm{b}}$ & $1.20 ; 1.12$ & $1.41 ; 1.98$ & $1.33 ; 1.69$ \\
\hline
\end{tabular}

a Values for plasma cholesteryl esters are means $\pm \mathrm{SD}$ for five animals. $\mathrm{b}$ Values for cholesteryl esters bound to elastin are individual data for two samples. $* p<0.01$, $* * p<0.001$ vs. control group, $\uparrow p<0.01, \ddagger p<0.001$ vs. cholesterol-fed group.

tissue levels of triglyceride and phospholipids of both experimental rabbits were slightly higher than those in the controls.

The supernatants from the saline extraction and sequential collagenase and elastase digestions of the aortic tissues from the rabbits of the three groups were analyzed for cholesterol, uronic acid, and hydroxyproline as measures of lipoprotein, glycosaminoglycan, and collagen, respectively, and the results are shown in Table 3(A). On the basis of dry defatted tissue weight, the highest amount of cholesterol was removed with saline in all groups. In the Saline-extracted fraction, cholesterol contents were significantly higher in the cholesterol-fed and pantethinetreated groups than in the control, whereas uronic acid and hydroxyproline levels remained unchanged. Concentrations of cholesterol and uronic acid in the Collagenase-digested fraction were significantly increased in the cholesterol-fed group over the control values, while the hydroxyproline content did not differ. In this fraction three components in the aortic tissues from the pantethine-treated rabbits remained unchanged. In the Elastase-digested fraction, cholesterol contents were elevated 2.5-fold in the aortas from the atherogenic-diet group and twice in those from the pantethine-treated animals. In contrast, uronic acid and hydroxyproline concentrations remained unchanged. Cholesterol contents in these fractions were significantly $(p<0.01)$ reduced by pantethine treatment.

Cholesterol and collagen contents in the Collagen fraction, and cholesterol and elastin residues in the Elastin fraction are summarized in Table 3(B). In the Collagen fraction, cholesterol contents were significantly higher in the cholesterolfed animals than in the controls, while collagen, calculated from hydroxyproline content, was almost the same in the two groups. The Elastin fraction of the aortas from the cholesterol-fed rabbits yielded about three times more cholesterol than corresponding treatment of the aortas from the control animals. Dry weights of the defatted elastin, analyzed as a measure of elastin protein, tended to decrease in both experimental groups. Thus, cholesterol concentrations per unit of weight of collagen and elastin were significantly increased 2-3-fold in the cholesterol-fed rabbits. Treatment of the cholesterol-fed rabbits with pantethine resulted in significant and consistent reductions in cholesterol content and ratio of cholesterol to the macromolecules in the Elastin fraction.

To obtain further information concerning the accumulation of cholesterol on arterial elastin, we carried out gas-liquid chromatographic analyses to deter- 
mine the fatty acid compositions in cholesteryl ester fractions in the plasma and in the Elastin fraction of aortic tissues from the rabbits of the three groups. The ratios of oleic acid to linoleic acid in these cholesteryl ester fractions are tabulated in Table 4. The ratio in plasma cholesteryl esters from five control animals was $0.42 \pm 0.05$, and the relative proportions of these fatty acids to the total fatty acids in plasma cholesteryl esters were $20.2 \pm 2.1 \%$ for oleic acid and $47.8 \pm 2.7 \%$ for linoleic acid. On the other hand, the cholesteryl esters bound to the Elastin fraction from normal rabbit aortas had a very low proportion of linoleic acid, and the $18: 1 / 18: 2$ ratios was in the range of $1.1-1.2$. In addition to the increase in concentration, there were marked changes in the fatty acid composition of plasma cholesteryl esters in the experimental animals. The ratio of 18:1/18:2 in plasma cholesteryl esters was significantly $(p<0.001)$ increased in the experimental groups, whereas those in the Elastin fraction of aortic tissues were only slightly increased in these groups. Furthermore, these ratios in the plasma and Elastin fraction showed almost no change between the cholesterol-fed and pantethine-treated groups.

Thus, in the cholesterol-fed rabbits cholesterol and phospholipid concentrations in the plasma and cholesterol contents in all extracted fractions of the aortic tissues were highly significantly increased over those in the control animals. In contrast, triglyceride and free fatty acid concentrations in the plasma, and triglyceride, phospholipid, glycosaminoglycan, collagen and elastin contents in the aortas from the atherosclerotic rabbits were slightly higher than those in the controls. Treatment of the atherosclerotic rabbits with pantethine resulted in significant reductions in the concentrations of all components which were increased in the cholesterol-fed group in the plasma and in the aortic tissues.

\section{Histological findings}

No lipid accumulation and no lipid-laden foam cells were observed in the aortic tissues obtained from the control rabbits. In these animals, fine elastic fibers in a few layers were occasionally observed locally in the intima with a few intimal cells. However, the thickness of the intimal layer never exceeded $10 \mu \mathrm{m}$ in the control group of animals.

In contrast, rabbits fed the atherogenic-diet for six weeks showed diffuse intimal thickenings, mainly consisting of accumulated foam cells. The degree of intimal thickening was highest in the aortic arch region (mean $\pm \mathrm{SD}, 135 \pm 5 \mu \mathrm{m}$ ) and marked in the aorta under the diaphragm $(130 \pm 30 \mu \mathrm{m})$. Samples from one of the two rabbits in the cholesterol-fed group showed intimal thickening of similar extent in the four standard regions. In areas where foam cells had accumulated markedly, the foam cells in the deeper layer in the intima underwent necrosis and fine fat droplets were scattered around these cells. In the thickest lesion examined $(160 \mu \mathrm{m}$, under the diaphragm) smooth muscle cells had proliferated in the superficial layer of the intima and the majority of foam cells in the deep layer of intima were necrotic resulting in abundant extracellular lipid accumulation (Fig. 3). Although fine elastic fibers and Azan stain-positive fibers were usually 


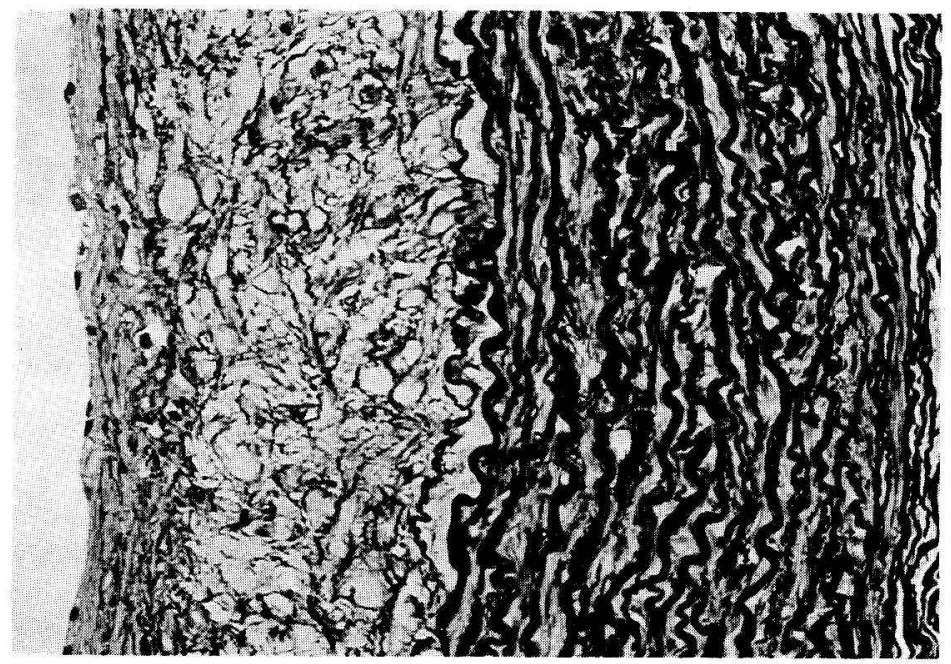

Fig. 3. Histological appearance of the abdominal aorta from a rabbit of the cholesterolfed group, illustrating an atherosclerotic intimal plaque consisting of fibromuscular proliferation in the subendothelium and a large amount of extracellular lipid accumulation in the depth. Weigert-Masson trichrome stain, $\times 280$.

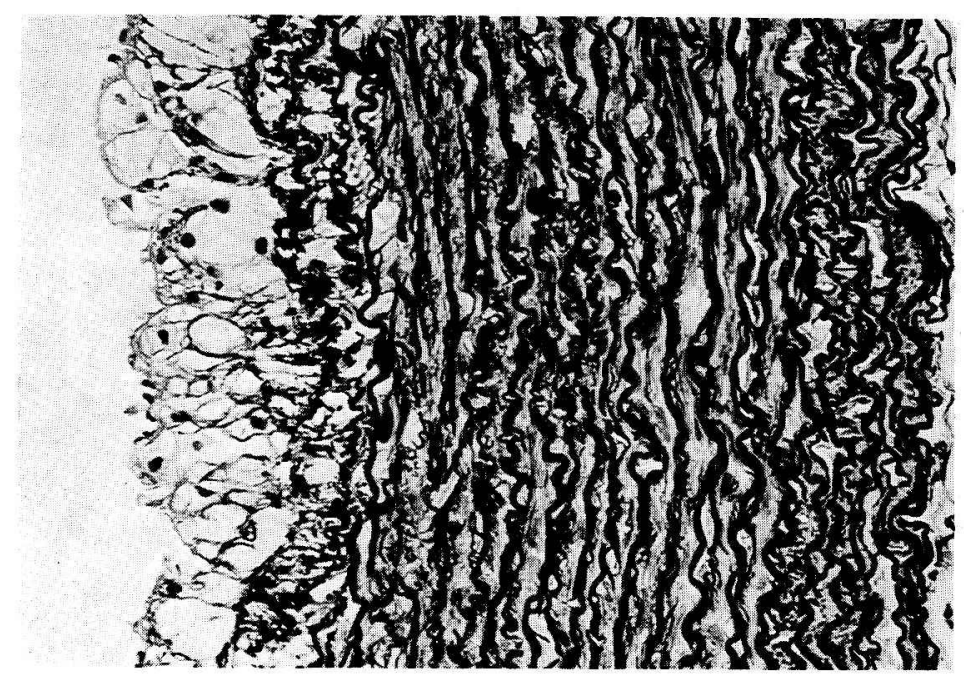

Fig. 4. Histological appearance of the aortic arch from a rabbit of the cholesterol-fed/pantethine-treated group, illustrating from cell lesion with neither remarkable fibromuscular proliferation nor atheromatous foci. Weigert-Masson trichrome stain, $\times 280$.

observed surrounding foam cells, when these foam cells began to disintegrate, the fibers and the interstitial ground substances containing acid mucopolysaccharides rapidly increased. Focal lipid accumulation in the inner and middle layers of the media was noted together with severe lipid deposition in the intima. Smooth 
muscle cells disappeared zonally or spotted in the inner layer of the media and were replaced with the increasing ground substances on which fine fat droplets were closely deposited.

In the pantethine-treated rabbits, although the mode of lipid accumulation in the aortic wall was the same as that in the cholesterol-fed group, its degree was remarkably mild compared with the atherosclerotic animals. Although the intimal thickenings in the regions of the aortic arch and abdominal aorta were observed in both rabbits examined in this group, the degree of these thickenings were low, being $47.5 \pm 37.5$ and $30.0 \pm 10.0 \mu \mathrm{m}$, respectively. In addition, no lesion was observed in the upper and lower thoracic aorta of one rabbit in this group. The intimal lesions in the pantethine-treated rabbits also consisted of the accumulated foci of foam cells as those in the cholesterol-fed animals. However, we were unable to detect disintegration of foam cells or intense subendothelial proliferation of smooth muscle cells covering foam cell foci in this group (Fig. 4). Mild fibro-cellular thickening of the intima $(20 \mu \mathrm{m})$ without lipid deposition was observed in this experimental group. In the intima, fine elastic laminae had proliferated in a few layers, smooth muscle cells were observed surrounding these elastic laminae, and alcian blue-positive ground substances were moderately increased.

\section{DISCUSSION}

The present study was designed primarily to investigate the distribution of complexes between lipoproteins and connective tissue macromolecules in the early stage of lipid accumulation in the arterial wall in an experimental model. Although cholesterol feeding of rabbits has been used commonly as a model of experimental atherosclerosis, relatively little is known about the biochemical composition of the cholesterol-induced atherosclerotic arterial wall. We investigated the distribution of cholesterol in the aortas from the normal, atherosclerotic, and pantethine-treated atherosclerotic rabbits by extracting and analyzing the complexes between lipids and glycosaminoglycan, collagen, and elastin.

No significant difference in body or tissue weights was observed either interor intra-group in the present study with use of SPF New Zealand white rabbits. We observed, however, that the SPF rabbits had a wide range of plasma cholesterol concentrations and that they showed a variation in their cholesterolemic response to cholesterol feeding. From this point of view, our original aim of minimizing variation was only partly achieved in the present study.

At the tissue lipid level, there were slight rises in triglyceride and phospholipid contents in the atherosclerotic aortas, but cholesterol, especially the esterified form, was markedly and significantly elevated. Furthermore, the levels of cholesterol bound to glycosaminoglycan, collagen, and elastin were highly significantly increased in the atherogenic-diet group over those in the control animals. The contents of cholesterol in both the normal and atherosclerotic aortas were the 
highest in the part bound to glycosaminoglycans, whereas the increases in cholesterol content due to cholesterol feeding were most significant in the part bound to elastin.

With the use of various extraction procedures, the interaction of lipoproteins and glycosaminoglycans was studied in detail by Srinivasan et al. [18] in the aortas of normal and atherosclerotic rabbits. They concluded that the presence of glycosaminoglycan along with lipoproteins in the various extracted fractions and in the ultracentrifugal fractions indicated that all materials solubilized by these procedures were lipoprotein-glycosaminoglycan complexes. However, the elevation in the cholesterol content of atherosclerotic aortas was found to be greater than that in uronic acid in the Elastase-digested fraction from the cholesterol-fed rabbits both in their and our present experiments. From the results found for the Elastasedigested fraction and Elastin fraction, complexes of lipids with elastin, in addition to those with glycosaminoglycans, were suggested to be increased in the aortas of atherosclerotic animals. These results suggest the importance of complex formation of lipoproteins with elastin in the lipid accumulation in atherosclerotic lesions [12].

The relative proportion of linoleic acid in plasma cholesteryl esters was significantly higher than that of oleic acid in the normal rabbits. On the contrary, the cholesteryl esters bound to the Elastin fraction from normal rabbit aortas had a very low proportion of linoleic acid and a high proportion of oleic acid. These results suggest that the cholesteryl esters bound to connective tissue macromolecules in the normal rabbit aortas were derived from the vascular cells, in which lipoprotein-cholesterol entered via receptor-mediated endocytosis and was re-esterified with oleic acid by the acyl coenzyme A: cholesterol acyltransferase reaction [3]. On the other hand, there were marked increases in the relative proportion of oleic acid and in the ratio of oleic acid/linoleic acid in the plasma cholesteryl esters from the cholesterol-fed rabbits. In spite of the significant increase in content, however, no increase was observed in the ratios in cholesteryl esters bound to the Elastin fraction from the atherosclerotic rabbits. Furthermore, the ratios in the plasma and in the Elastin fraction showed no significant change in the cholesterol-fed animals. These results suggest that the cholesteryl esters accumulated on the connective tissue macromolecules in the atherosclerotic rabbits may be derived from the plasma directly, in addition to the cellular cholesteryl esters. Studies in our laboratory $[6,11,12]$ have indicated the in vivo and in vitro formation of stable complexes between plasma LDL and human arterial elastin.

The glycosaminoglycan content in the atherosclerotic aortas from the cholesterol-fed rabbits $(9.72 \pm 0.92 \mathrm{mg}$ uronic acid/g dry tissue) was slightly but significantly different from that from the normal $(7.69 \pm 0.46 \mathrm{mg} / \mathrm{g})$, whereas in the pantethine-treated animals $(8.66 \pm 0.76 \mathrm{mg} / \mathrm{g})$ it remained unchanged. There were no consistent changes in collagen and elastin contents in the aortas from either atherosclerotic group. These results are in agreement with those of Ehrhart and 
Holderbaum [19], who observed increases in collagen and elastin biosynthesis and no change in their contents per unit of dry defatted weight in the aortas from cholesterol-fed rabbits.

Treatment with pantethine significantly reduced the increased levels of cholesterol in the aortic tissues from the cholesterol-fed rabbits. These reductions in cholesterol contents by pantethine were observed in all fractions extracted from the aortic tissues. Pantethine, a derivative of pantothenic acid and an intermediate precursor of coenzyme $\mathrm{A}$, has been shown to have an interesting hypolipidemic activity in experimental animals [7, 20, 21] and in human beings [9, 22]. In the present study the lipid-lowering effect of this drug was confirmed at the end of the 6-week cholesterol feeding period. However, no difference in plasma cholesterol concentration was found between the cholesterol-fed and pantethine-treated rabbits for up to 4 weeks. These small differences in plasma cholesterol concentrations could not account for the large decreases in cholesterol content in the aortic tissues from the pantethine-treated animals. To support the evidence, data from two aortas, C-2 and P-3, from two rabbits, one from each experimental group, are shown in Table 5. Although almost the same level of plasma cholesterol was found in these rabbits, the lipid content of aortic tissue (P-3) from the pantethine-treated

Table 5. Plasma cholesterol levels and contents of various components of aortas from a cholesterol-fed rabbit or from a cholesterol-fed/pantethine-treated one.

\begin{tabular}{|c|c|c|}
\hline & Cholesterol-fed & Pantethine-treated \\
\hline Rabbit & $\mathrm{C}-2$ & P-3 \\
\hline Plasma cholesterol $(\mathrm{mg} / 100 \mathrm{ml})$ & 1,068 & $1,0.92$ \\
\hline Aortic tissues & & $\mathrm{mg} / \mathrm{g}$ dry tissue \\
\hline Cholesterol & 14.5 & 10.0 \\
\hline Triglyceride & 12.7 & 12.8 \\
\hline Phospholipids & 38.4 & 30.8 \\
\hline \multicolumn{3}{|l|}{ Saline-extracted fraction } \\
\hline Cholestero! & 6.74 & 4.54 \\
\hline Uronic acid & 4.95 & 5.01 \\
\hline Hydroxyproline & 0.53 & 0.51 \\
\hline \multicolumn{3}{|l|}{ Collagenase-digested fraction } \\
\hline Cholesterol & 2.00 & 1.12 \\
\hline Uronic acid & 2.24 & 2.09 \\
\hline Hydroxyproline & 39.0 & 34.9 \\
\hline \multicolumn{3}{|l|}{ Elastase-digested fraction } \\
\hline Cholesterol & 5.90 & 4.09 \\
\hline Uronic acid & 2.02 & 2.11 \\
\hline Hydroxyproline & 6.90 & 6.19 \\
\hline \multicolumn{3}{|l|}{ Collagen fraction } \\
\hline Cholesterol & 4.91 & 4.11 \\
\hline Collagen & 239 & 246 \\
\hline \multicolumn{3}{|l|}{ Elastin fraction } \\
\hline Cholestero! & 3.39 & 1.46 \\
\hline Elastin & 397 & 420 \\
\hline
\end{tabular}


rabbit was considerably lower than that from the cholesterol-fed animals (C-2). One possible reason for these observations may be that the drug directly acts on the aortic tissues, for it reportedly increases the cholesteryl ester hydrolase activity in homogenates of rat arterial tissues [21] and causes a decrease in the biosynthesis of cholesterol in human skin fibroblasts [8]. The histological results in the present study also support this speculation.

With respect to morphological findings, intimal lipid depositions caused by a cholesterol-rich diet are characterized by focal accumulation of foam cells. Atheromatous changes with many extracellular lipid depositions develop in areas where marked formation and disintegration of foam cells are observed. Moreover, intimal lesions occassionally involve the atherosclerotic plaques with abundant proliferation of smooth muscle cells. Similar histological observations in the aortic wall were noted in the present experiment using SPF rabbits fed an atherogenicdiet containing $0.5 \%$ cholesterol and $5 \%$ peanut oil for six weeks. Apparent reductions in the severity of atherosclerotic intimal lesions in rabbits receiving cholesterol with pantethine were observed, and no extracellular lipid accumulation derived from the disintegration of foam cells and no marked proliferation of smooth muscle cells were noted in these rabbits.

Clinical studies have shown that oral administration of pantethine moderately decreases plasma LDL-cholesterol and increases HDL-cholesterol levels [9, 22]. Furthermore, pantethine has been shown by in vitro experiments to act directly on the vascular smooth muscle cells, to increase the number of lysosome [23], to elevate the activities of acid cholesteryl ester hydrolase [21] and acid phosphatase [23] in the intimal cells, and to inhibit the cell-impairing action of hyperlipidemic sera or homocysteine (unpublished data). These actions of pantethine seem to prevent the accumulation of intracellular lipids in foam cells and smooth muscle cells, and to inhibit the disintegration of these cells.

In the cholesterol-fed rabbits, smooth muscle cells proliferated markedly and existed in a form of thick layer in the superficial part of the intima in areas which were characterized by significant accumulation of foam cells. It seems to be attributable to the remarkable cellular proliferative reactions of arterial wall, because intimal lesions were markedly severe in this group.

In the pantethine-treated rabbits, mild fibrocellular intimal thickenings with scarce lipid deposition were found in the aortic wall. Possible reasons for this observation may be that the scar-like intimal thickening is formed after disappearance of foam cell foci, and that intense proliferation of smooth muscle cells in the intima is inhibited by the elevation of plasma HDL levels and suppression of the formation of foam cells due to the pantethine treatment.

This study was supported in part by a grant from Daiichi Seiyaku Co., Ltd., Tokyo, Japan. The authors gratefully acknowledged the technical assistance and valuable discussions of Drs. H. Okabe and T. Sakurada, Messrs. M. Kita and M. Kusanagi. 


\section{REFERENCES}

1. Woodard, J.F., Srinivasan, S.R., Zimmy, M.L., Radhakrishnamurthy, B., and Berenson, G.S. (1976): Electron microscopic feature of lipoprotein-glycosaminoglycan complexes from human atherosclerotic plaque. Lab. Invest., 34, 516-521.

2. Srinivasan, S.R., Yost, K., Radhakrishnamurthy, B., Dalferes, E.R., Jr., and Berenson, G.S. (1980): Lipoprotein-hyaluronate association in human aorta fibrous lesions. Atherosclerosis, 36, 25-37.

3. Smith, E.B., and Smith, R.H. (1976): Early changes in aortic intima, in Atherosclerosis Reviews, Vol. 1, ed. by Paoletti, R. and Gotto, A.M.,Jr., Raven Press, New York, pp. 119136.

4. Kramsch, D.M., Franzblau, C., and Hollander, W. (1971): The protein and lipid composition of arterial elastin and its relationship to lipid accumulation in the atherosclerotic plaque. J. Clin. Invest., 50, 1666-1677.

5. Tokita, K., Kanno, K., and Ikeda, K. (1977): Elastin subfraction as binding site for lipids. Atherosclerosis, 28, 111-119.

6. Noma, A., Takahashi, T., Yamada, K., and Wada, T. (1979): Elastin-lipid interation in the arterial wall. Part 1. Extraction of elastin from human aortic intima. Atherosclerosis, 33, 29-39.

7. Tomikawa, M., Nakayasu, T., Tawara, K., Kameda, K., and Abiko, Y. (1982): Effect of pantethine on lipoprotein profiles and HDL subfractions in experimentally hypercholesterolemic rabbits. Atherosclerosis, 41, 267-277.

8. Ranganathan, S., Jackson, R.L., and Harmony, J.A.K. (1982): Effect of pantethine on the biosynthesis of cholesterol in human skin fibroblasts. Atherosclerosis, 44, 261-273.

9. Gaddi, A., Descovich, G.C., Noseda, G., Fragiacomo, C., Colombo, L., Craveri, A., Montanari, G., and Sirtori, C.R. (1984): Controlled evaluation of pantethine, a natural hypolipidemic compound, in patients with different forms of hyperlipoproteinemia. Atherosclerosis, 50, 73-83.

10. Srinivasan, S.R., Radhakrishnamurthy, B., Dalferes, E.R., Jr., and Berenson, G.S. (1979): Collagenase-solubilized lipoprotein-glycosaminoglycan complexes of human aortic fibrous plaque lesions. Atherosclerosis, 34, 105-118.

11. Noma, A., Takahashi, T., and Wada, T. (1981): Elastin-lipid interaction in the arterial wall. Part 2. In vitro binding of lipoprotein-lipids to arterial elastin and the inhibitory effect of HDL on the process. Atherosclerosis, 38, 373-382.

12. Noma, A., Hirayama, T., and Yachi, A. (1983): Studies on the binding of plasma low density lipoproteins to arterial elastin. Connect. Tissue Res., 11, 123-133.

13. Okabe, H., Uji, Y., Nagashima, K., and Noma, A. (1980): Enzymic determination of free fatty acids in serum. Clin. Chem., 26, 1540-1543.

14. Bartlett, G.R. (1959): Phosphorus assay in column chromatography. J. Biol. Chem., 234, 466-468.

15. Bitter, T., and Muir, H.M. (1962): A modified uronic acid carbazole reaction. Anal. Biochem., 4, 330-334.

16. Fujishima, T., Okabe, H., and Noma, A. (1975): The determination of hydroxyproline in urine. Jpn. J. Clin. Pathol., 23, 815-818 (in Japanese).

17. Bergman, I., and Loxley, R. (1970): The determination of hydroxyproline in urine hydrolysates. Clin. Chim. Acta, 27, 347-349.

18. Srinivasan, S.R., Yost, K., Bhandaru, R.R., Radhakrishnamurthy, B., and Berenson, G.S. (1982): Lipoprotein-glycosaminoglycan interactions in aortas of rabbits fed atherogenic diets containing different fats. Atherosclerosis, 43, 289-301. 
19. Ehrhart, L.A., and Holderbaum, D. (1980): Aortic collagen, elastin and non-fibrous protein synthesis in rabbits fed cholesterol and peanut oil. Atherosclerosis, 37, 423--432.

20. Carrara, P., Matturri, L., Galbussera, M., Lovati, M.R., Franceschini, G., and Sirtori, C.R. (1984): Pantethine reduces plasma cholesterol and the severity of arterial lesions in experimental hypercholesterolemic rabbits. Atherosclerosis, 53, 255-264.

21. Shinomiya, M., Matsuoka, N., Shirai, K., Morisaki, N., Sasaki, N., Murano, S., Saito, Y., and Kumagai, A. (1980): Effect of pantethine on cholesterol ester metabolism in rat arterial wall. Atherosclerosis, 36, 75-80.

22. Itakura, H., Murase, T., Akanuma, Y., and Kosaka, K. (1980): Increase of high density lipoprotein with pantethine observed more than one year and on the mechanism of its action. Abstract of VII Int. Symposium Drug Affect Lipid Metabolism, p. 20.

23. Yoshida, Y., Mitsumata, M., Fukuda, T., and Ooneda, G. (1983): Beneficial effects of pantethine on regression of atherosclerosis. Abstract of VIII Int. Symposium Drug Affect Lipid Metabolism, p. 76. 